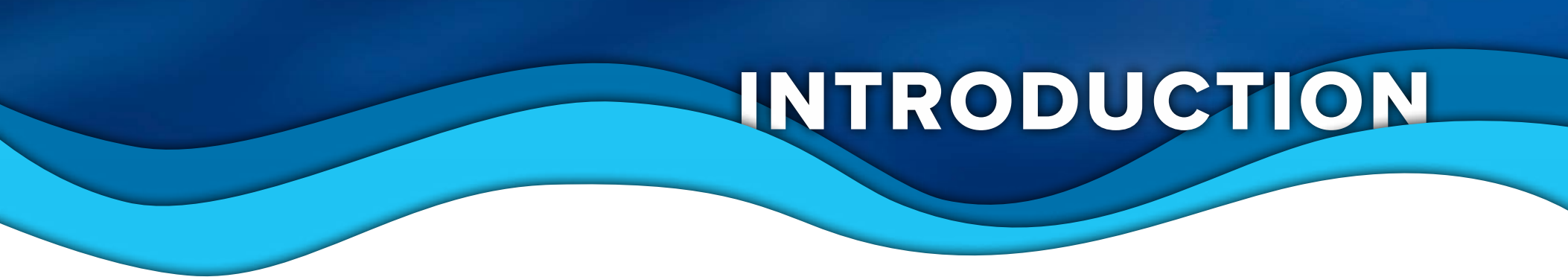

\title{
Introduction to the Ocean Observing Supplement to Oceanography
}

By Ellen S. Kappel, S. Kim Juniper, Sophie Seeyave, Emily A. Smith, and Martin Visbeck

Scientists observe the ocean's complex and interwoven physical, chemical, biological, and geological processes to understand the numerous ways in which the ocean sustains life and provides benefits to society, and to forecast events that affect humankind and the planet. They use a range of instruments to gather data, from simple nets and thermometers to sophisticated sensors aboard autonomous vehicles that transmit data back to laboratories nearly instantaneously. Some instruments are tethered to ships or moored to the seafloor, and others drift with ocean currents, move autonomously, or are controlled from land. There are also specialized satellites, aircraft, and drones that carry ocean observing sensors. Observations are made over hours to days to years in all parts of the global ocean, from the tropics to the poles, from the coasts to the open ocean, and from the seafloor to its surface waters.

The many different types of ocean observations allow scientists to detect and track pollutants and toxic substances such as oil slicks, plastics, and other marine debris; to document ocean warming and acidification as well as changes in ocean circulation and ecosystem health; and to better forecast hazards such as hurricanes, earthquakes, tsunamis, ocean heatwaves, flooding, and harmful algal blooms.

In this supplement to the December issue of Oceanography, we introduce frontiers in ocean observingthe articles describe new technologies and reveal some exciting results that advance our understanding of the world ocean and its resources and support its sustainable use and management. For this 2021 inaugural supplement, potential authors were invited to submit letters of interest aligned with the priorities of the UN Decade of Ocean Science for Sustainable Development (2021-2030) in the following topical areas:

TOPIC 1. Ocean-Climate Nexus. Observations related to climate monitoring, modeling, and forecasting; sea level rise; and ocean acidification.
TOPIC 2. Ecosystems and Their Diversity. Studies and observations for habitat mapping and restoration and for biodiversity monitoring, in particular, the relationship between biodiversity and climate change, as well as applications for natural resource management and conservation.

TOPIC 3. Ocean Resources and the Economy Under Changing Environmental Conditions. Observations and services in support of the blue economy (e.g., energy, transport, tourism), sustainable use of ocean resources (e.g., fisheries/aquaculture, genetic resources, minerals, sand), and marine spatial planning.

TOPIC 4. Pollutants and Contaminants and Their Potential Impacts on Human Health and Ecosystems. Systems for monitoring pollutants/contaminants (e.g., heavy metals, nutrients, plastics, and organic pollutants, as well as noise) and their dispersal, and potential links to policy frameworks.

TOPIC 5. Multi-Hazard Warning Systems. Observing systems and information services supporting disaster risk reduction and improving human health, safety, and food security.

We received 127 letters of interest from the global ocean observing community, from which we chose the subset of articles contained in this supplement. For many of the articles, we asked authors who had never before worked together to collaborate and submit one combined article. We also chose a few articles to close the supplement with descriptions of exciting new ocean observing technologies.

We thank Ocean Networks Canada, the US National Oceanic and Atmospheric Administration's Global Ocean Monitoring and Observing Program, the international Partnership for Observation of the Global Ocean, and the US Arctic Research Commission for generously supporting publication of this Ocean Observing supplement.

ARTICLE DOI: https://doi.org/10.5670/oceanog.2021.supplement.02-01 


\section{AUTHORS}

Ellen S. Kappel (ekappel@geo-prose.com), Geosciences Professional Services Inc., USA. S. Kim Juniper, Ocean Networks Canada (ONC). Sophie Seeyave, Partnership for Observation of the Global Ocean (POGO), Plymouth Marine Laboratory, UK.

Emily A. Smith, National Oceanic and Atmospheric Administration, Global Ocean Monitoring and Observing Program (NOAA/GOMO), USA. Martin Visbeck, GEOMAR Helmholtz Centre for Ocean Research Kiel, Germany.

\section{ARTICLE CITATION}

Kappel, E.S., S.K. Juniper, S. Seeyave, E.A. Smith, and M. Visbeck. 2021. Introduction to the ocean observing supplement to Oceanography. P. 1 in Frontiers in Ocean Observing: Documenting Ecosystems, Understanding Environmental Changes,

Forecasting Hazards. E.S. Kappel, S.K. Juniper, S. Seeyave, E. Smith, and M. Visbeck, eds, A Supplement to Oceanography 34(4), https://doi.org/10.5670/oceanog.2021. supplement.02-01.

\section{COPYRIGHT \& USAGE}

This is an open access article made available under the terms of the Creative Commons Attribution 4.0 International License (https://creativecommons.org/ licenses/by/4.0/), which permits use, sharing, adaptation, distribution, and reproduction in any medium or format as long as users cite the materials appropriately, provide a link to the Creative Commons license, and indicate the changes that were made to the original content. 